\title{
Los jóvenes y el deporte en la política pública de los Centros Educativos Comunitarios
}

\author{
Young people and sport \\ in Community Education Centers' public policy
}

\section{Andrea Quiroga Galeazzi*}

\footnotetext{
* Docente del Instituto Superior de Educación Física, Universidad de la República. Participante de la línea de investigación «Deporte, cultura y política» del Grupo de Estudios Sociales y Culturales del Deporte. Licenciada en Educación Física (ISEF-UdelaR). Maestranda en Deporte (UNLP).

$\triangle$ aquirogaleazzi@gmail.com http://orcid.org/0000-00021880-9348
}

RECIBIDO: 31.8.2021

\section{Resumen}

En el presente trabajo se expone cómo la política pública de los Centros Educativos Comunitarios (CEC) construye y entiende a los jóvenes, para ponerlo en diálogo con las conceptualizaciones sobre deporte como fenómeno social y cultural. Esto permite discutir cómo esta política colabora con las representaciones y los sentidos que en los CEC se le dan al deporte y los retroalimenta. Los CEC son un programa de una de las instituciones educativas públicas del Uruguay, el Consejo de Educación Técnico Profesional (CETP), que se implementa en lugares de contexto socioeconómico correspondiente a los dos primeros quintiles de ingreso, con el fin de acercar el sistema educativo tradicional a los y las jóvenes que no han podido acceder a él luego de finalizar la escuela primaria. La mirada sobre la juventud es una construcción social a partir de la cual se piensan, en este caso, políticas públicas y su implementación. Para este trabajo es importante establecer cuál es la mirada sobre las y los jóvenes que surge tanto de los documentos oficiales como de las y los actores que forman parte de los CEC.

Palabras clave: joven, deporte, política gubernamental, Uruguay. 


\section{Abstract}

Community Education Centers, a CETP's (Council of Technical Professional Education) public program, are implemented in places of a socioeconomic context corresponding to the first and second income quintile. CECs were designed for young people who, after finishing primary school, were not able to access secondary education, to bring them closer to the traditional education system. This paper analyses how this public policy builds youth and understands young people, in dialogue with the conceptualizations of sport as a social and cultural phenomenon, making it possible to discuss the view of this public policy and to what extent it collaborates with or feeds back into the representations and meanings given to sport in the CECs. Youth is understood as a social construction from which, in this case, public policies and their implementation are conceived. This paper focusses on the importance of establishing the view on young people both from the perspective of official documents as well as from the CECs actors.

Keywords: youth, sport, government policy, Uruguay.

\section{Introducción}

Los Centros Educativos Comunitarios (CEC) funcionan como un proyecto de una de las instituciones educativas públicas de Uruguay, el Consejo de Educación Técnico Profesional - Universidad del Trabajo del Uruguay (CETP-UTU), desde 2014. Apunta a los y las adolescentes a quienes se les dificulta sostener los cursos del sistema educativo tradicional. Están ubicados en barrios periféricos de Montevideo: Casabó, La Teja, Bella Italia y Casavalle.

En su propuesta curricular incluyen siete asignaturas: tres de áreas tecnológicas (Robótica, Informática y Audiovisual), tres de áreas transversales (Matemática, Lengua y Filosofía) y una del área Deporte y Recreación. En esta última se trabaja con todo el estudiantado los viernes, coordinando actividades con la comunidad, y de lunes a jueves sin horario específico, con diferentes actividades deportivo-recreativas.

Trabajé como docente del área Deporte y Recreación entre 2014 y 2018. Allí tomé la decisión de que este proyecto fuera el tema de mi tesis de maestría, cuyo objetivo general es analizar los procesos de legitimación de la educación física y el deporte en los CEC, dado que allí el rol docente también implica un trabajo conjunto con el coordinador del CEC, cuidar que los y las estudiantes no salgan de clase y ocuparse del grupo en los tiempos libres. Esto, que extiende la tarea más allá de la enseñanza específica de técnicas, tácticas y reglamentos, me dificultaba el diseño de las clases, ya que no tenía herramientas para pensar en el abordaje de cuestiones por fuera de lo instrumental. En este artículo presentaré un avance de dicha investigación referido a cómo es la mirada de la 
política pública de los CEC y qué incidencias tiene allí el deporte, con la intención de fortalecer la producción que se viene haciendo en la región en cuanto a pensar en el deporte por fuera de su lógica tradicional.

\section{Antecedentes}

Antes de comenzar con las discusiones particulares de esta política pública, creo necesario presentar algunos estudios que ya se han realizado sobre el deporte y la inclusión social en la región. El texto de Guedes et al. (2006) «Projetos sociais esportivos: notas de pesquisa» surge de una investigación que se llevó adelante en un curso de formación permanente de emprendedores de ONG donde se discutió si en los proyectos se dan o no principios sociales y culturales sobre deporte. Se plantea la naturalización del valor pedagógico que tiene la práctica deportiva, que establece un vínculo con determinada perspectiva de la sociedad y con algunos agentes sociales. Concluyen que es necesario - y también hay interés en ello- incluir las condiciones sociales y culturales en los estudios sobre estos proyectos deportivos orientados a jóvenes y niños de los quintiles 1 y 2 , para complementar su educación formal.

Levoratti (2016) presenta Deporte y política socio-educativa. Una etnografía sobre funcionarios y profesores de Educación Física, producto de la investigación de su tesis de maestría. Allí discute sobre las representaciones y prácticas sobre deporte de profesores de Educación Física y funcionarios, en programas de la política socioeducativa en la provincia de Buenos Aires (2004-2011). En los diversos discursos sobre la incorporación del deporte en la política socioeducativa identifica la consideración del deporte como una herramienta para la inclusión social, que favorece la reducción de la violencia, del consumo de drogas y de problemas sociales. En sus conclusiones se observan dos perspectivas sobre el deporte: por un lado, pensar este campo como arena pública donde se verifican diferentes tensiones sociales; por otro, darle un sentido salvador, como herramienta que todo lo puede, ya que posibilita adquirir valores, normas, reglas. Busca demostrar así que el deporte funciona como una esfera de la vida social de los actores, por lo que genera.

En Uruguay se llevó adelante el trabajo Identificación de potencialidades inclusivas en los proyectos sociales deportivos (PSD) de Malvín Norte (Levoratti y Vales, 2020), donde se analizaron las potencialidades inclusivas de los PSD. Se buscaba analizar las estrategias de inclusión social en los PSD y construir un modelo en el que se evidenciaran las potencialidades encontradas para que pudiera adaptarse a diferentes proyectos. 


\section{Einfoque metodológico}

Realicé la investigación sobre los CEC de Bella Italia y Casabó. El objetivo general fue analizar los procesos de legitimación de la educación física y el deporte en los CEC pertenecientes al CETP. De forma exploratoria, con entrevistas en profundidad y grupos focales, busqué identificar las principales representaciones que tienen docentes, funcionarios, responsables del Departamento de Cultura Física del CETP e inspectores de los CEC sobre la educación física y el deporte en esta experiencia, así como explorar el rol asignado a las prácticas deportivas en la propuesta educativa de los CEC. Entrevisté a las autoridades creadoras del proyecto, a coordinadoras de los CEC de Bella Italia y Casabó, a docentes del área Deporte y Recreación de todos los CEC, y trabajé con grupos focales de docentes de las áreas transversales y tecnológicas.

El enfoque metodológico que utilicé fue la etnografía. Para ello me basé en Guber (2001), que me permitió comprender que se necesita elaborar una representación coherente sobre lo que las autoridades de estos CEC piensan y dicen sobre la asignatura Deporte y Recreación. Por eso, la tesis busca comprender la legitimidad que tiene el deporte en las autoridades creadoras e impulsoras del proyecto. Para esta búsqueda, y basándome en Guber (2001), entiendo importante posicionarme en un lugar que suponga ignorancia, de modo de poder identificar lo exótico en dichos espacios, ya que desde otro lugar no sería posible acercarse a las representaciones sobre el deporte que estas personas tienen.

Para el avance que presento en este artículo utilicé parte de las entrevistas y uno de los documentos que es específico del área, Propuesta de cultura física para los CEC 2016 (Consejo de Educación Técnico Profesional [CETP], 2016).

\section{Cómo afecta el deporte a la política pública de los CEC}

El discurso futbolístico no requiere de una competencia vaga, general, sino de una forma restringida y concentrada. El fútbol, con su arena y sus actores, permite asumir posiciones, expresar opiniones y proponer soluciones. El fútbol es como un discurso político. Es para el adulto masculino un juego pedagógico que enseña a mantener un lugar que le pertenece. (Archetti, 1984, p. 83)

Para pensar la política pública de los CEC y su relación con la juventud, es importante, como plantea Reguillo Cruz (2007), trabajar en una caracterización que problematice lo que se ve de ella para así comprender lo que sucede. Una dificultad es que el 
pienso de esas políticas parte de una caracterización hecha por personas externas que no problematizan lo que allí ven para entender lo que sucede, sino que buscan su modificación, intentando acercarlas a las estructuras propias de la sociedad y las instituciones.

Si asociamos esta mirada con el rol que tenía el deporte en las public schools, podemos encontrar cierta relación, dado que las prácticas deportivas allí son creadas y pensadas para disminuir la violencia en los y las jóvenes, y disciplinarlos para ir acercándolos a las estructuras propias de la vida social y civilizada correspondiente a un burgués (Elias y Dunning, 1992).

El deporte entra allí a cumplir este rol, a colaborar, en cierta forma, con la «adjudicación» de figuraciones necesarias para que la juventud logre el objetivo del mundo adulto. A su vez, se puede considerar que la implementación de estas prácticas deportivas colabora con los aspectos sociales y de conducta que plantean Elias y Dunning (1992): las reglas, a partir de las cuales comienzan a estructurarse ciertas pautas de conducta y a limitar lo que la sociedad puede o no sentir; el tiempo, herramienta que antes no existía y que también comienza a estructurar la forma de vivir de la sociedad, ya que su vida empieza a girar en torno a un tiempo que no es real y del cual todas las personas dependen de manera universal; la individualización, el sentid o de lo propio y la lucha por llegar a lograr algo por uno mismo; el autocontrol, que será una característica fundamental para la burguesía en ese momento y hoy en día para toda persona joven que quiera (y deba) insertarse en el sistema educativo formal. Para el caso de los CEC:

Nuestros gurises no son cualquier gurí, entonces ellos necesitan moverse un poco de... Son chiquilines que hace dos o tres años que no estudian, por ejemplo; entonces, a un chiquilín que hace dos o tres años que no estudia, tenerlo cuatro horas sentado es complejo; necesita ese espacio de movimiento [...] Me parece que sí, que todos los días es necesario un ratito; se mueven, vienen cansados [...] Sin ese espacio... el cuerpo es todo un tema porque, claro, la cabeza no piensa si el cuerpo no puede estar quieto, y tenemos muchos chiquilines que no pueden estar quietos, entonces es un momento de correr, de gastar energía, de saltar, de pensar... (Leticia Silva, comunicación personal, 2018) ${ }^{1}$

Se puede identificar aquí esta cuestión planteada por Reguillo Cruz (2007), en la que se piensa una forma de trabajar y concebir la política pública y la implementación del deporte a partir de allí. En esta concepción no se piensa en sus propios jóvenes, sino que se toma cierta forma de trabajo considerada tradicionalmente como la adecuada y se la pone en funcionamiento, esperando el mismo resultado. 
En contraposición, se puede pensar el deporte como arena pública cuya implicancia en parte refiere a la construcción de identidad que ocurre en las prácticas deportivas, como plantea Archetti (1984). Esa construcción está dada por quienes trabajan y conforman esas prácticas; por lo tanto, se entiende que allí son escuchadas todas las voces, siempre y cuando se trabaje desde el deporte con esta perspectiva. Sin embargo, este enfoque no garantiza que estas cuestiones sucedan constantemente y construyan determinadas estructuras. El hecho es que muchas veces no se debate, discute o dialoga sobre ellas, lo cual genera y reproduce estructuras que tal vez se contradicen, tanto con la política pública a partir de la cual trabajamos como con nuestros propios fines allí.

A la hora de pensar el deporte es necesario tener en cuenta que «por debajo de esas similaridades existe un mundo de diferencias y variaciones que es necesario describir» (Archetti, 1984, p. 74). En el caso de los CEC, es preciso identificar estas diferencias y variaciones para trabajar sobre ellas, porque ellas también construyen determinada identidad. Como plantea Archetti (1984) en su texto Fútbol y ethos, al ver a las sociedades desde el mundo simbólico que se construye alrededor del deporte, se pueden identificar ciertos aspectos de la cultura y del ethos nacional. Entonces se podría decir que, en función de cómo vemos —en este caso, desde la política públicay enseñamos el deporte, será el mundo simbólico que se construya a su alrededor.

\section{Representaciones sobre la juventud}

En el caso de los CEC, en su proyecto borrador, elaborado en 2014 y sin modificaciones hasta la fecha, se plantea un interés por trabajar con «formatos que favorezcan la materialización del derecho a la educación y que se adecuen a las características del desarrollo de estos jóvenes en concordancia con sus necesidades y posibilidades reales» (Proyecto borrador, 2014, p. 3). Para ello se presentan espacios que tendrán como base principal el desarrollo de las y los jóvenes, buscando construir en ellos una visión sobre el valor que tiene «prosperar y comprender integralmente el mundo que habitan» (CETP, 2014, p. 3). El objetivo general que aquí se formula busca el desarrollo de la juventud potenciando los espacios que tengan que ver con «la integración social, la formación del carácter, la convivencia, la creatividad y el pensamiento crítico, así como las habilidades y capacidades relacionadas con el trabajo en equipo, la resolución de problemas, la generación de proyectos, la comunicación» (CETP, 2014, p. 5).

Con base en Chavez (2005), se puede identificar en este discurso una representación del joven «como un ser incompleto» (p. 15), idea asignada por el adulto, que establece un derecho para poder intervenir sobre él, ayudarlo a completar su ser. En 
este sentido, podría decirse que no hay posibilidad de sujeto político en los y las jóvenes. A su vez, sobre estos objetivos deben ser pensadas las clases y las formas de trabajo de todas las asignaturas. Desde Deporte y Recreación se puede interpretar que se piensa en el deporte como el lugar a partir del cual se puede comunicar a los y las jóvenes la forma de ver el mundo y los valores que deben tener. Parecería verse aquí una afirmación que también trae Archetti (1984) cuando plantea que el fútbol «es una manera de reproducir ciertas lealtades primordiales» (p. 78). La cuestión aquí es: ¿cuál es esa forma?, ¿hay valores propios del deporte que permiten esto que la política pública espera?

Tanto en la fundamentación como en el objetivo general del proyecto (objetivos específicos no figuran), se considera un punto de partida en el que los y las jóvenes no tienen ninguna de estas cuestiones nombradas, lo cual se puede relacionar con «el reconocimiento explícito de no ser portadores de ninguna verdad absoluta» (Reguillo Cruz, 2007, p. 14). A su vez, son cuestiones relacionadas con la moral y no con lo académico, por la que lo que se busca y la mirada desde la que se parte se orientan a lograr que la juventud forme parte de espacios con determinada estructura que exigen determinados códigos sociales.

Esta visión también se puede encontrar en la entrevista realizada a uno de los referentes institucionales:

Con J. J. unos vecinos nos dicen: «Estamos deseando que empiecen, así los gurises dejan de salir a la calle» [...] Visualizar que un montón de gurises que andaban en la calle sin hacer nada, que no trabajaban, que no estudiaban, se volverían a reencontrar con las ganas de aprender... (Horacio Pérez, comunicación personal, 2018).

Deporte y Recreación trabaja como primer aspecto la convivencia; cabe aclarar que es la única asignatura a la que se le adjudica este propósito. Se puede entender que es su única responsabilidad, ya que en la descripción no aparece ningún contenido de los que se podrían considerar propios del área.

Se puede observar además otra representación que plantea Chavez (2005), referida al joven como un futuro peligro al que hay que cuidar para que no vaya por ese camino. Sin embargo, en los documentos no aparece una problematización de los espacios que habitan los y las jóvenes fuera de las estructuras socializadoras. No tener en cuenta esos espacios donde están y se manifiestan, trabajar en pro de su adaptación a determinadas instituciones o estructuras de la sociedad sin pensar, problematizar o discutir sobre eso lleva a no tener en cuenta que en esos espacios hay «confrontación, producción y circulación de saberes, que se traduce en acciones» (Reguillo Cruz, 2007, p. 14).

Es aquí donde comienzo a ver la relación de esta política pública con la producción de un sentido sobre el deporte. Si entendemos el deporte como un fenómeno social y 
cultural en el que se pueden identificar diversas tensiones para problematizar y debatir, entonces, en el accionar de los y las jóvenes, y a partir de diferentes prácticas deportivas, puede discutirse sobre lo que allí sucede y, así, llegar a entender las representaciones que tienen en sus diversas acciones políticas, las cuales no dejan de serlo por estar fuera de una estructura institucional o social. Sin embargo, este no es el enfoque con el que se piensa y trabaja el deporte. En las entrevistas que realicé también se advierte esta relación entre la mirada hacia la juventud desde la política pública y su vínculo con el deporte cuando se plantea que «el concepto que estaba ubicado en el CEC era de deporte, no educación física, porque en realidad se entendía que ese espacio fuera un espacio de socialización y desarrollo de algunas capacidades a las que nos parecía que el deporte podía sumar» (Rosana Flores, comunicación personal, 2019).

La posibilidad de socialización y desarrollo vincular a partir del deporte se puede considerar como herramienta que permite llegar a otras cosas. Esto es propio de un enfoque sobre el deporte del que Cagigal (1979) es uno de sus referentes, que busca, como se observa en el proyecto borrador, un desarrollo de las habilidades para estar en sociedad y formar parte de las diversas estructuras sociales:

Apostábamos más que nada, además del deporte, no tanto a lo técnico, sino a los aspectos más sociales, de compañerismo. No competitivo; lo competitivo era lo que menos nos importaba, porque eso generaba muchos roces también entre ellos. De vez en cuando hacer una competencia sana estaba bueno, porque los motivaba de otra manera, pero más que nada [apostábamos] a lo social y al compañerismo, a cooperar con el otro para poder llegar a un determinado objetivo, a esos valores. (Carolina Paz, comunicación personal, 2018)

A partir de esta cita se puede pensar sobre el desplazamiento que tiene el deporte dentro de cada sociedad y cómo colabora en «toda forma clásica de constitución de sujetos para transformarse en una ideología en el sentido althusseriano» (Alabarces, 1998, p. 5). Es decir, se asimila al aparato ideológico que reproduce y sostiene el sistema en el que estamos, aunque se considere que solo se toman de él sus «buenos valores»; sin que el deporte sea parte del Estado, pero sí teniendo instituciones que fomentan y sostienen la ideología del sistema económico capitalista y de competencia; considerando que, eliminados lo técnico y la competencia, ya nos alejamos de esta ideología.

A partir de este proyecto borrador y del inicio de las actividades en los CEC, el departamento de Cultura Física correspondiente al CETP crea una Propuesta de Cultura Física para los CEC. En ella se plantea un rol docente que es bastante similar a lo que el proyecto presenta como perfil docente, que incluye dos características: 
«ser disparadores de proyectos, poderlos coordinar con el resto del colectivo docente y ponerlos en práctica. Como algo específico del área se fomentará el trabajo en equipo» (CETP, 2016, p. 1). El programa y las unidades de trabajo que plantea son las siguientes:

Programa y unidades de trabajo de la Propuesta de Cultura Física para los CEC

\begin{tabular}{|c|c|c|}
\hline Unidad de Recreación & Unidad de Deporte & $\begin{array}{l}\text { Unidad de Prevención } \\
\text { Cuidado y Salud }\end{array}$ \\
\hline $\begin{array}{l}\text { Trabajo en hábitos de convi- } \\
\text { vencia, integración, coopera- } \\
\text { ción y colaboración. } \\
\text { Juegos sociales, predeporti- } \\
\text { vos, cooperativos y grandes } \\
\text { juegos (ejemplo: yincana). } \\
\text { Construcción de juegos } \\
\text { a partir de materiales } \\
\text { reciclables. } \\
\text { Actividades de expresión } \\
\text { artística. } \\
\text { Habilidades circenses. } \\
\text { Campamentos. }\end{array}$ & $\begin{array}{l}\text { Trabajo en equipo. } \\
\text { Deportes sociomotores } \\
\text { y psicomotores, cerrados } \\
\text { o abiertos. } \\
\text { Trabajo de la lógica depor- } \\
\text { tiva a través del predeporte, } \\
\text { juegos aplicativos y juegos } \\
\text { reglados. } \\
\text { Reglas de los deportes dados. } \\
\text { Deporte adaptado. } \\
\text { Formación corporal. } \\
\text { Desarrollo de las habilidades } \\
\text { motoras básicas. }\end{array}$ & $\begin{array}{l}\text { Aplicación de la unidad que } \\
\text { propone cultura física para } \\
\text { todos sus cursos, basada } \\
\text { en dos pilares: reanimación } \\
\text { cardíaca y reflexiones sobre } \\
\text { los consumos adictivos. } \\
\text { Análisis de los hábitos salu- } \\
\text { dables del ejercicio físico y } \\
\text { la alimentación. }\end{array}$ \\
\hline
\end{tabular}

Fuente: CETP (2016, p. 2).

\section{El deporte y la juventud}

Definir el deporte no es algo sencillo, sobre todo por las diferentes formas que toma en función del contexto, la historia y su forma de producirse y reproducirse. A su vez, al entenderlo como un fenómeno social y cultural, pertenece a la arena pública. Al decir de Archetti (1998), es un espacio donde salen a la luz tensiones y dramas de la sociedad. Decir esto no implica que lo que allí suceda es un reflejo de lo que sucede en la sociedad. Por el contrario, entender el deporte como una arena pública es entenderlo como parte de la sociedad en la que se practica. Por lo tanto, las prácticas deportivas cobran forma y sentido a partir del espacio cultural, social e histórico donde se llevan a cabo. Esto implica que en una misma arena —en este caso el deporte- se podrán identificar diferentes significados y visiones en función del lugar donde uno se encuentre.

Un claro ejemplo de esto lo trae Archetti (1984) cuando analiza el fútbol como arena pública en Argentina y en Brasil. En Argentina, a partir de las hinchadas se puede identificar una construcción de masculinidad necesaria e identitaria para ellas, mientras 
que en Brasil lo que cuenta es el jogo bonito, y en las hinchadas se observa una lucha más referida a la creatividad que a la masculinidad. ¿Por qué esto es así? Porque la mirada del deporte como arena pública visibiliza estas cuestiones, nos permite identificar las representaciones que se producen en determinadas prácticas, y ver esto nos permite trabajar más allá de lo técnico, lo táctico y lo estratégico, pero, sobre todo, trabajar sobre las construcciones que a partir de nuestra enseñanza sobre el deporte producimos, reproducimos o fortalecemos.

Así se puede asimilar el planteo de Brownell (2019) sobre «la parcialidad de observar al deporte como un sistema de "características formales-estructurales"» (p. 235), donde se deja entrever que los deportes y sus formas de practicarlo tienen que ver con una interacción social. Esto hace que sus prácticas y reglamentos estén en constante discusión y negociación. Los cambios que vayan a suceder allí tienen que ver con las relaciones de poder que estén establecidas entre quienes forman parte de dicha negociación.

Esto quiere decir que cada grupo o clase que practica determinado deporte le dará cierto sentido y significado a dicha práctica, construyendo así identidades que tienen cierta función y fin en el espacio donde se dan. Pero también serán muy importantes las decisiones que se tomen sobre qué formas de deporte se trabajarán y desde qué perspectiva. Esto permite que allí emerjan cuestiones que hacen a dicha práctica deportiva, pero que también tienen estrecha relación con el lugar y el momento histórico en el que se está dando. Por tanto, no es posible que esas prácticas sean neutrales, cuestión bastante naturalizada.

Si el deporte que se practica tiene relación directa con un tiempo, una historia, un espacio y sujetos que lo construyen y deconstruyen, analizan y discuten sobre sus reglas y prácticas, entonces no es posible que sea apolítico. De hecho, que el deporte se considere apolítico es una postura política, la cual lleva a reproducir un discurso hegemónico de cuya existencia muchas veces no somos conscientes, pero que al reproducirlo seguimos fortaleciendo y, por tanto, legitimando -incluso si dejamos por fuera de esto las condiciones históricas y sociales del deporte, su ritmo, evolución y crisis, cuestiones que para Bourdieu (1990) es necesario analizar porque entiende que el deporte es un fenómeno social moderno con una historia relativamente autónoma.

Esto puede coincidir con las críticas que Brownell (2019) le hace al texto de Guttman (1978) From ritual to record. The Nature of Modern Sports. Si entendemos que el deporte tiene autonomía relativa en lo que refiere a su historia, entonces podemos entender las tensiones que presenta Brownell (2019). Como se ha planteado, ya desde la introducción del deporte en las public schools este comienza a separarse y hacerse neutral respecto del mundo social. ¿Con qué fin? Que los y las jóvenes, pertenecientes a las elites de la burguesía, es decir, la gentry inglesa que allí concurría - personas ricas y bien educadas, pero no nobles (Elias y Dunning, 1992)—, adquirieran, a través del 
cuerpo, el gusto «por la actividad gratuita, dimensión fundamental del ethos de las "élites burguesas"» (Bourdieu, 1990, p. 197).

Aquí se puede identificar un indicio de dicha legitimidad sobre el deporte desde lo apolítico y, por tanto, neutral, dado que el capital cultural y simbólico que lo constituye gira en torno a ello, a que lo beneficioso de las prácticas deportivas es que nos unen, que disminuyen las desigualdades. Pero Archetti (1984) en sus escritos ya expresa esta idea y sostiene que «el fútbol aparece como el mundo de la igualdad y de la justicia, y como tal ejerce una fascinación especial en los sectores populares y subordinados» (p. 88). Lo que allí se reproduce es una concepción del deporte como panacea, la posibilidad de los sectores populares de romper con la lógica del capitalismo y llegar a un lugar inesperado. La cuestión aquí es: ¿para cuántos de esos sectores esto es una realidad?, ¿cuántos de ellos, en el afán de llegar a ese lugar tan deseado, en vez de romper con las jerarquías de la sociedad, terminan fortaleciéndolas cada vez más?

Esta concepción del deporte como neutral también puede observarse en el texto de Brownell (2019), donde se presentan los intentos por llevar a Oriente las prácticas y culturas occidentales. Tal forma de entender el deporte como neutral y «limpio» de toda cultura, sin un anclaje territorial que dé cuenta la sociedad donde se reproduce, fue lo que llevó a estos occidentales a intentar forzar la introducción del deporte con determinadas características y formas de practicarlo que no se correspondían con el lugar donde se intentaba implantar.

Los educadores de la YMCA estaban tratando de crear buenos ciudadanos para la democracia inculcando el respeto por el juego limpio, pero los alumnos habían crecido bajo una dinastía imperial en la cual la moralidad social consistía en conocer el principio de $l i$, lo que significaba mostrar respeto adecuado por la propia apariencia y la del otro. (Brownell, 2019, p. 235)

Entender así el deporte implica verlo como una integralidad, donde no hay partes internas o externas a investigar o estudiar por separado, sino que cada práctica deportiva se construye y construye determinada identidad a partir de las y los actores que la conforman. Con esto me refiero a todo lo que forma parte de su mercado y su significación, tanto su práctica como los consumos deportivos. Esta cuestión está relacionada con los y las jóvenes; Reguillo (2007) trae la identificación que de ellos hace la sociedad como sujetos de consumo; por lo tanto, es una temática que podría pensarse para tensionar y reflexionar.

Muchas veces no tenemos en cuenta este consumo a la hora de pensar el deporte (ni a la juventud) como objeto, o al pensar una política pública que esté basada en él. Esta característica que se le adjudica como constructor de identidad no quiere decir que cada práctica deportiva construye sentidos y significados por separado del resto, sino 
que se pone en disputa cierto capital allí producido y se lucha por él, sea este simbólico, económico, cultural o lingüístico (Bourdieu, 1990). De esta forma, pueden identificarse determinadas prácticas en ciertos lugares y con ciertas formas, mientras en otros sitios existen otras.

Dentro de la propuesta del Departamento de Cultura Física se puede observar una perspectiva desde la teoría de Parlebas. Creo importante analizar por qué la toman, ya que hace una división entre el adentro y el afuera de las prácticas; el adentro contiene las acciones motrices y cómo los elementos funcionales modifican, o no, dichas acciones, lo que deja en el afuera toda la trama social y cultural. Sin embargo, Saraví (2007) plantea que existe una mala interpretación sobre la teoría de Parlebas, ya que en ella explicita que su puesta en funcionamientos sin un estudio previo adecuado conduce al consumo de una forma de enseñanza. Las interpretaciones sobre esta teoría han llevado a entender que son cuestiones totalmente distintas, lo cual entra en contradicción con lo planteado por autores como Archetti (1984) y Brownell (2019), quienes entienden que a estas acciones motrices que la teoría de Parlebas presenta en un adentro se llegó, se llega y se seguirá llegando de formas que tienen que ver con un momento en la historia social que condujo a determinadas personas a alcanzar acuerdos y negociaciones reglamentarias y sobre la puesta en práctica de los deportes que hizo que estas acciones motrices sean las adecuadas. Pero ¿qué sucedería si en estos ámbitos de negociación y análisis hubiera un cambio en quién tiene el poder para tomar estas decisiones y modificar cuestiones que influyen directamente en estas acciones? ¿No habría modificaciones en ellas?

Por otro lado, se continúa viendo la misma posición ya presentada, en la que el o la joven está allí para aprender todo lo que su docente tiene para ofrecerle sobre las habilidades necesarias para comportarse y estar en sitios estructurados e institucionales. A su vez, se adjudican una serie de contenidos correspondientes al área, pero que no dan cuenta de una intención de diálogo, reflexión y un trabajo en pro de entender de dónde parten los y las jóvenes y qué cuestiones pueden problematizarse y trabajarse a partir de dicha tensión.

Hay una coincidencia entre el interés del departamento de Cultura Física en trabajar sobre los consumos adictivos y el planteo de Reguillo Cruz (2007) sobre la idea de los jóvenes «como "responsables" de la violencia en las ciudades» (p. 20), hecho que se identifica con el consumo y las drogas. Por lo tanto, comienza a construirse una idea de las y los jóvenes como delincuentes y personas violentas, representación que coincide con la presentada por Chavez del joven como desviado o posible peligro, cuyo gran manipulador es la droga y para quien el deporte podría ser una posibilidad de salvación. Cabe aclarar que Reguillo Cruz (2007) no plantea que la juventud sea responsable ni la relaciona con las drogas, sino que en su trabajo plantea las construcciones sociales que hay sobre ella, entre las que prevalece la del consumo. Al igual que en los CEC, tal como comenta una entrevistada: 
Yo no soy la indicada para hablar de que el deporte es salud y esas cuestiones, pero en verdad los libera de poder estar con un porro en la boca, ¿entendés? 0 pensar que a la noche se van a juntar para tomarse esto o fumar aquello... (Paula Calvo, comunicación personal, 2018)

Se puede identificar aquí la idea de que el deporte podría disolver la problemática del consumo, ya que se le adjudican determinados valores que generan la creencia de que, por el solo hecho de estar practicándolo, estas tensiones propias de la juventud, que son construidas en un entorno histórico, social y cultural al cual dan respuesta, desaparecerán. Sin embargo, a partir del enfoque planteado anteriormente se puede decir que esto no sucede por el simple hecho de practicar deporte, sino que sucederá por la tensión que se ponga a estas situaciones, su explicitación y debate junto con los y las jóvenes. Eso permitirá convertir la interpretación de que cualquier joven viene aquí a ser amoldado a cierta estructura y salvado de lo que le hace mal por el simple hecho de estar donde está y ser lo que es, en una identificación del lugar que ocupa en la sociedad, reconociendo las cuestiones que produce y reproduce con sus prácticas. Pero podemos compartir con Trejo (2013) que «La práctica del fútbol no es una herramienta automática. No se trata de una solución milagrosa. No hay que creer en un discurso único mediante el cual todo es bello y extraordinario» (p. 125).

Entender a los y las jóvenes como sujetos de consumo es categorizarlos de manera universal y a su vez asumir que dicha categoría tiene cierta neutralidad de la que no es posible escapar. Así como ya se aludió a la imposibilidad de un deporte apolítico, la mirada sobre la juventud cumple con la misma conceptualización: «las categorías no son neutras, ni aluden a esencias; son productivas, hacen cosas, dan cuenta de la manera en que diversas sociedades perciben y valoran el mundo y, con ello, a ciertos actores sociales» (Reguillo Cruz, 2007, p. 29). Por eso es de gran importancia distinguir cuál es la mirada que se tiene de la juventud a partir de esta política pública, ya que ella es la estructura de todo lo que deviene en este proyecto.

\section{Consideraciones finales}

Tanto en la política pública como en los discursos de las personas entrevistadas, se puede identificar una representación del deporte como salvador, como algo que todo lo puede. No hay un cuestionamiento o un análisis orientado a llevar adelante una enseñanza del deporte que posibilite esta «salvación» fuera del disciplinamiento por repetición y mandato directo. Podemos decir que esta perspectiva, más que colaborar en la construcción de jóvenes ciudadanas y ciudadanos reflexivos y críticos de la forma de 
vida que quieran llevar, los introduce dentro del funcionamiento social capitalista profundamente y de manera exitosa.

Un punto de fuga posible para pensar el deporte es planteado por Archetti (1984) cuando intenta vincular la visión del mundo con el ethos, aspectos que conforman las prácticas deportivas y que deben tenerse en cuenta en el momento en que se considera al deporte como parte de la cultura, ya que en estos se combinan lo cognitivo con el afecto y los estilos. Todas estas cuestiones se construyen y también reproducen en las prácticas deportivas.

En esa dirección, entender el fútbol como parte de una cultura implica que los actores sociales con su comportamiento concreto, sus discursos, sus gritos, sus indignaciones y sus muestras de satisfacción tratan de poner cierto orden, de reproducirlo o de cambiarlo. (Archetti, 1984, p. 77)

Pensar desde aquí el deporte nos puede permitir, en primer lugar, el diálogo con los y las jóvenes y la identificación de sus necesidades, intereses y representaciones sobre las prácticas que ofrecemos o presentamos. Y, a partir de allí, la construcción de diálogo y reflexión que se genere sobre el deporte será característica de ese grupo y habilitará diálogos y debates sobre dramas que sean parte de sus realidades, lo cual permitiría estipular reglas y significados a partir de sus formas de vida y no de las del mundo adulto. Esta identificación también puede abrir el debate sobre la posibilidad o no de formar parte de ese mundo adulto y qué implicancias eso tiene.

\section{Referencias bibliográficas}

Alabarces, P. (1998). ¿De qué hablamos cuando hablamos de deporte? Nueva Sociedad, (154), 74-86.

Archetti, E. (1984). Fútbol y ethos. Buenos Aires: Flacso. (Monografías e informes de investigación, 7).

Archetti, E. (1998). Prólogo. En P. Alabarces, R. Di Giano y J. Frydenberg (comps.), Deporte y sociedad (pp. 9-12). Buenos Aires: Eudeba.

Bourdieu, P. (1990). Sociología y cultura. México: Grijalbo.

Brownell, S. (2019). Los problemas con la teoría del ritual y la modernización y por qué necesitamos a Marx: Un comentario sobre From Ritual to Record. En P. Scharagrodsky y C. Torres (comps), El rostro cambiante del deporte: Perspectivas historiográficas angloparlantes 1970-2010. Buenos Aires: Prometeo.

Cagigal, J. M. (1979). Cultura intelectual y cultura física. Buenos Aires: Kapelusz. 
Chavez, M. (2005). Juventud negada y negativizada: Representaciones y formaciones discursivas vigentes en la Argentina contemporánea. Última Década, 13(23), 9-32.

Consejo de Educación Técnico Profesional. (2014). Proyecto borrador: Centros Educativos Comunitarios. (Manuscrito inédito).

Consejo de Educación Técnico Profesional. (2016). Propuesta de cultura física para los CEC 2016. (Manuscrito inédito).

Elias, N., y Dunning, E. (1992). Deporte y ocio en el proceso de la civilización. Madrid: Fondo de Cultura Económica.

Guber, R. (2001). La etnografía: Método, campo y reflexividad. Buenos Aires: Siglo XXI.

Guedes, S. L., D’Anglo Davies, J., Antunes Rodrigues, M., y Medeiros Santos, R. (2006). Projetos sociais esportivos: Notas de pesquisa. Ponencia presentada en Usos do passado, $12 .^{\circ}$ Encuentro Regional de Historia.

Levoratti, A. (2016). Deporte y política socio-educativa: Una etnografía sobre funcionarios y profesores de educación física. Buenos Aires: Prometeo.

Levoratti, A., y Vales, L. (2020). Identificación de potencialidades inclusivas en los proyectos sociales deportivos de Malvín Norte. (Manuscrito inédito).

Reguillo Cruz, R. (2007). Emergencia de culturas juveniles. Bogotá: Norma.

Saraví, J. R. (2007). Praxiología motriz: Un debate pendiente. Educación Física y Ciencia, 9, 103-117.

Trejo, F. (2013). A ball can change the world. Percepciones y situaciones en el mundial de los desamparados, la Homeless World Cup: Una revisión crítica desde la vivencia y la mirada de los jugadores. Acta Sociológica, 60, 103-128. 